\title{
Efecto del valor agregado del consumo cultural: una aproximación a la economía naranja en Colombia
}

Fecha de recepción: 28 de agosto de 2017

Fecha de aprobación: 07 de noviembre de 2017

Pp. 4 - 11

Cómo citar este artículo: Jiménez, I. F.; Millán, M. F. y Suárez, D. F. (2017). Efecto del valor agregado del consumo cultural: una aproximación a la economía naranja en Colombia. Revista Ploutos 7(2), 4-11.

\section{Resumen}

La economía naranja es definida como el conjunto de actividades que de manera encadenada permiten que las ideas se transformen en bienes y servicios creativos, cuyo valor está determinado por su contenido de propiedad intelectual. El valor estimado de esta economía es de alrededor de 4,3 billones de dólares. La determinación de su valor en Colombia es el objetivo de esta investigación; aun así, se hace un cálculo aproximado dada la complejidad de su valoración. Para ello se escogieron un grupo de nueve de actividades de consumo cultural durante un periodo de 10 años; artes visuales, artes escénicas, educación cultural, creación literaria, musical, teatral y audiovisual, filmes y videocintas, fabricación de juegos y juguetería, música, editorial y creación publicitaria. Se estima que en Colombia entre el 2005 y el 2014 la economía naranja, en promedio, correspondió a 0,69 \% del PIB, su valor agregado sobre la producción al 53,09\%, y su grado de industrialización al 0,36 \%.

Palabras clave: economía naranja, consumo cultural, valor agregado, Colombia.

\section{The effect of the added value of cultural consumption: an approach to the orange economy in Colombia}

\section{Abstract}

The orange economy is defined as the set of activities that, in a chained way, allow ideas to be transformed into creative goods and services, whose value is determined by their content of intellectual property. The estimated value of this economy is around 4.3 trillion dollars. The objective of this investigation is to determine its value in Colombia; though, an approximate calculation is made given the complexity of its valuation. For that purpose, a group of nine cultural consumption activities was chosen over a period of 10 years: visual arts, performing arts, cultural education, literary creation, musical creation, theatrical and audiovisual creation, films and videotapes, toys and games manufacturing, music, publishing, and advertising creation. It is estimated that between 2005 and 2014, the Orange Economy in Colombia, on average, corresponded to 0.69 \%of the Gross Domestic Product (GDP), its added value over production to $53.09 \%$, and its degree of industrialization to $0.36 \%$.

Key words: orange economy, cultural consumption, added value, Colombia. 


\section{Effets de la valeur ajoutée de la consommation culturelle: une approche de l'économie orange colombienne}

Résumé

L'économie orange peut se définir comme l'ensemble des activités permettant que les idées se transforment en biens et services créatifs dont la valeur est déterminée par le contenu de propriété intellectuelle. La valeur estimée de cette économie représente environ 4,3 milliards de dollars. L'objectif de cette étude est de déterminer une estimation de la valeur de cette économie en Colombie. Nous avons ainsi choisi un groupe de neuf activités de consommation culturelle étudié sur une période de 10 ans: arts visuels, arts scéniques, éducation culturelle, création littéraire, musicale, théâtrale et audiovisuelle. Selon certaines estimations, l'économie orange colombienne correspondait entre 2005 et 2014 à environ 0,69 \% du PIB, sa valeur ajoutée sur la production à 53,09 \%, et son degré d'industrialisation à 0,36\%.

Mots-clés: économie orange, consommation culturelle, valeur ajoutée, Colombie.

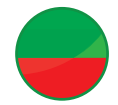

\section{A geração de valor compartilhado em entidades do setor público: estudo de caso em uma entidade distrital de Bogotá}

\section{Resumo}

A economia laranja é definida como o conjunto de atividades que de maneira sequencial permitem que as ideias se transformem em bens e serviços criativos, cujo valor está determinado por seu conteúdo de propriedade intelectual. O valor estimado desta economia é de aproximadamente 4,3 bilhões de dólares. A determinação de seu valor na Colômbia é o objetivo desta pesquisa; ainda assim, faz-se um cálculo aproximado dada a complexidade de sua avaliação. Para isso se escolheu um grupo de nove atividades de consumo cultural durante um período de 10 anos; artes visuais, artes cênicas, educação cultural, criação literária, musical, teatral e audiovisual, filme e vídeos, fabricação de jogos e brinquedos, música, editorial e criação publicitária. Estima-se que na Colômbia entre 2005 e 2014 a economia laranja, em média, correspondeu a 0,69\% do PIB, seu valor agregado sobre a produção correspondeu a 53,09\%, e seu grau de industrialização correspondeu a 0,36\%.

Palavras-chave: economia laranja, consumo cultural, valor agregado, Colômbia. 


\section{Introducción}

En los últimos años, la literatura económica ha empezado a guiar sus intereses en estudiar de manera profunda un sector que ha generado transformaciones en la economía y que se ha convertido en promotor para la generación de nuevos empleos: la economía naranja, sector que se basa en el desarrollo/producción de contenidos culturales. Alrededor de la literatura internacional, se encuentran estudios e investigaciones que abordan este sector como uno de los potenciales a largo plazo. En el desarrollo de esta investigación, se pretende abordar la economía naranja, llevando a cabo un acercamiento a su definición actual y evidenciar su desarrollo y evolución histórica para el caso colombiano.

Con el desarrollo de un marco teórico, la investigación busca brindar un acercamiento a la economía naranja, posteriormente se realiza un proceso descriptivo de los subsectores que se encuentran ligados a ella en Colombia, y así establecer cuál es su contribución a la producción total del país. Así mismo, se analizan variables, como valor agregado, consumo cultural y producción total según la Cuenta Satélite de Cultura 2005-2016 del Departamento Administrativo Nacional de Estadística (DANE).

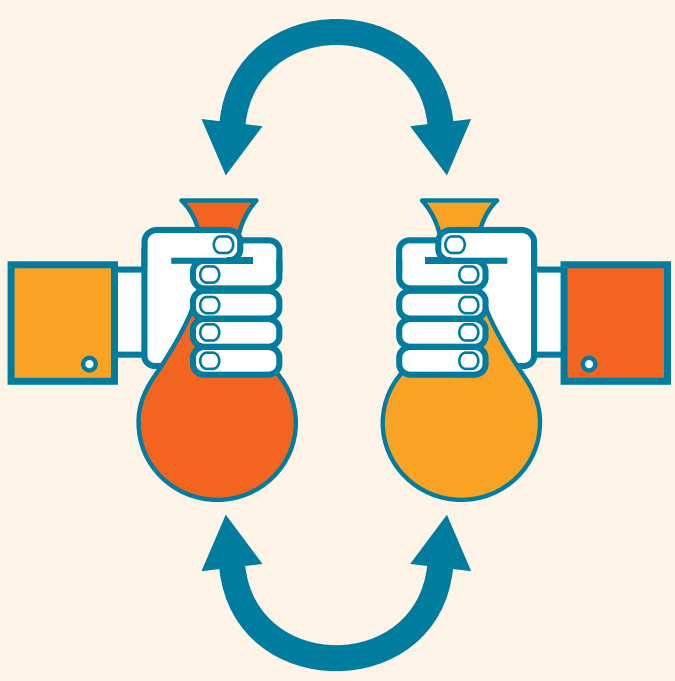

\section{Marco teórico}

El concepto de industria cultural ha evolucionado en los últimos años, y se ha convertido en un componente de bastante valor en una economía. Las actividades relacionadas han tomado relevancia, y los Gobiernos en sus políticas de desarrollo sectorial apuntan a aumentar sus fuerzas e inversiones, con el objetivo de potencializarlas. En la actualidad, la industria cultural, con la variedad de sus elementos, ha pasado a ser descrita como economía naranja, en la cual se genera una dinámica de producir, reproducir y difundir productos/servicios, que en ocasiones, resultan intangibles pero que generan experiencias de dualidad cultural y utilidades manteniendo un marco regulatorio (Szpibarg y Saferstein, 2014).

La economía naranja es definida por Valcerde, Salazar, Hernández y Ramírez (2015) como el conjunto de actividades que de manera encadenada permite que las ideas se transformen en bienes y servicios creativos, cuyo valor está determinado por su contenido de propiedad intelectual. Si estableciéramos la economía naranja como un país, tendría el tamaño equivalente a la cuarta economía más grande del mundo. La primera sería los Estados Unidos, con USD 15 billones; la segunda, China, con USD 7.3 billones; la tercera, Japón, con USD 5.9 billones; y la cuarta, la economía naranja, con USD 4.3 billones. América Latina sería la séptima economía más grande de la región, con un valor de USD 17500 millones. Colombia estaría ubicada en cuarto lugar, con USD 335000 millones.

Los países en América Latina han comenzado a considerar este sector como una oportunidad para dinamizar sus economías, como es el caso de Argentina donde la industria audiovisual, subsector de la economía naranja, se ha convertido en potencial exportador de contenidos audiovisuales, como novelas, programas de televisión, cortometrajes, largometrajes, etc., que se producen bajo parámetros estandarizados, y se encuentran siendo vendidos a diversos países y generan ingresos en la economía interna (Pis Diez y García, 2014).

Las exportaciones de economía naranja se encuentran bajo el nombre de bienes y servicios creativos, con un valor de USD 646000 millones, que ocupa el quinto rubro, solo por debajo de combustibles minerales y petróleo, equipos eléctricos y electrónicos, maquinaria, reactores nucleares, plantas térmicas y vehículos. En Centroamérica, 
las exportaciones de bienes creativos se clasifican en cerca de 50 $\%$ en diseño, $20 \%$ en materiales impresos, $10 \%$ en manufacturas artísticas, y el restante en artes visuales, medios de comunicación y audiovisuales (Quartesan, Romis y Lanzafame, 2007).

Diversos países han realizado esfuerzos por estudiar la economía naranja y medir su contribución estadísticamente. Los países europeos manifiestan la presencia de la industria cultural en la formulación de políticas locales que permitan contribuir al crecimiento de este sector. Aguiar (2014) establece que cada vez más la innovación es un componente determinante para el desarrollo de los sectores económicos y que, para el caso de España, la industria cultural manifiesta un nivel de innovación cercano a la media total de todos los indicadores de crecimiento económico. Se destaca que este sector manifiesta limitantes a la hora de innovar por factores de coste; principalmente, aquellas empresas pequeñas y medianas que describen la falta de fondos y de financiación.

La información para determinar estadísticamente la economía naranja en un país tiene muchos sesgos, dada la innovación del término; aun así, algunos autores han tratado de hacer cálculos o determinar los rubros que pueden entrar en ella para hacer una aproximación económica. El sector cultural ha sido la manera más precisa para determinar su valor. La contribución del PIB de 1995 a actividades directas con el sector fue de cerca de $1.17 \%$, y para 2001 había caído a $0.99 \%$. En el caso actividades conexas, para 1999, era de $0.26 \%$, y para 2001, de $0.19 \%$ (Aguado, 2010). Si realizáramos la suma de estos dos sectores, encontraríamos que, para 2001, su valor sería de $2.01 \%$. La contribución al PIB del sector cultural para 2003 en Colombia estaría cerca de $1.6 \%$, siendo el cuarto después de Uruguay con $3.2 \%$, Argentina con $2.6 \%$ y Chile con $1.9 \%$.

Si se hace una comparación de los aportes del PIB del sector cultural con otros sectores, para 2005, la industria cultural pesaba $2.06 \%$; los hoteles y restaurantes $1.94 \%$, los productos químicos $2.13 \%$; y el sector educativo $1.41 \%$. Dentro del sector cultural, la categoría de mayor inmersión ha sido las artes escénicas, de las cuales se destacan el teatro, la danza y la música (Castro et al., 2006).
Una investigación, según datos de la Encuesta de Consumo Cultural - ECC 2008, que utilizó un modelo probit concluyó que los principales determinantes de la asistencia a las artes escénicas eran el nivel educativo y el estatus socioeconómico, de manera que un nivel educativo y un estatus socioeconómico más bajo implican una menor probabilidad de asistencia a las artes escénicas. El efecto marginal de poseer estudios de educación superior - $0.3034-$ es cinco veces mayor al de tan solo tener estudios de primaria -0.0572-. Visitar museos, conciertos, recitales y música en vivo es también una variable que aumenta la probabilidad de asistencia, con un efecto marginal de 0.1663 . Pertenecer a una clase social media y alta son determinantes estadísticamente significativos que influyen en la asistencia a las artes escénicas. Vivir en Antioquia y Bogotá tiene un efecto marginal de 0.1820 y 0.1681 , respectivamente, que son estadísticamente significativos, lo cual permite concluir que pertenecer a estas dos regiones aumenta la probabilidad de asistir a las artes escénicas (Aguado y Palma, 2012).

La industria cultural de la música en Colombia ha mostrado una baja participación en la economía del país y también en la industria manufacturera. La participación de las actividades de edición y producción musical fue de tan solo $0.5 \%$ del valor agregado manufacturero en 2006 (Zuleta y Jaramillo, 2009). La industria musical se caracteriza por ser intensiva en mano de obra, lo cual se refleja en la mayor participación del personal ocupado -0.08 \%- frente al valor agregado -0.05\%-. Además, la industria musical tiene un alto costo financiero; en 2006, los gastos bancarios representaron $2.8 \%$ de las ventas, los intereses sobre préstamos aproximadamente $18 \%$ de las ventas y los impuestos $1.4 \%$ de estas (Zuleta y Jaramillo, 2009). El principal problema que enfrenta esta industria, al igual que las otras industrias culturales es la piratería. Según la Federación Internacional de la Industria Fonográfica (IFPI), la piratería de materia musical se lleva a cabo principalmente a través de internet y en menor medida en formato físico. En cuanto al mercado internacional, las ventas minoritarias de Colombia en 2004 representaron $0.14 \%$ del mercado mundial y $5.1 \%$ del mercado latinoamericano, aunque con una variación negativa en la participación en las unidades vendidas tanto en el mercado mundial como en el mercado latinoamericano (Zuleta y Jaramillo, 2009). 
La industria editorial y de comunicación gráfica colombiana está ubicada principalmente en Bogotá, con $63 \%$ de las empresas, $17 \%$ y $12 \%$ de estas se ubican en Antioquia y el Valle del Cauca, respectivamente. $51 \%$ de las empresas son pequeñas empresas, $26 \%$ son medianas y $23 \%$ son grandes (Andigraf, 2013). Los informes de sostenibilidad indican que los principales retos de la industria editorial son la competitividad, el aumento del valor agregado de las empresas y la exploración de nuevos mercados. Según la Cuenta Satélite de Cultura 2005-2016, en 2013 la industria editorial presentó una contracción de la producción de $12.8 \%$. La producción de esta industria está influenciada por el comportamiento de la oferta de servicios de la industria alimenticia, de bebidas y el comercio principalmente, por lo cual una contracción de estas industrias tiene un efecto negativo en la producción de la industria editorial (Andigraf, 2013).

\section{Desarrollo}

La economía naranja tiene limitaciones estadísticas para su valoración, así que fue necesario buscar aproximaciones a su concepto original. Los cálculos realizados a continuación se hicieron según la Cuenta Satélite de Cultura 2005-2016. El consumo cultural está definido como «aquellas actividades humanas y sus manifestaciones cuya razón de ser consiste en la creación, producción, difusión, consumo y apropiación de contenidos simbólicos relacionados con las artes y el patrimonio» (Banco Central de la República Dominicana, 2015, p 2). Las actividades referidas a la economía naranja y tomadas en este trabajo fueron artes visuales, artes escénicas, educación cultural, creación literaria, musical, teatral y audiovisual, filmes y videocintas, fabricación de juegos y juguetería, música, editorial y creación publicitaria.

Para llegar a un cálculo mucho más detallado, se decidió trabajar en la producción, el consumo intermedio y el valor agregado de cada una de las industrias. La producción es desarrollada bajo la responsabilidad, el control y la gestión de una unidad institucional, la cual utiliza la mano de obra y los activos para transformar los bienes y servicios usados como insumos de otros bienes y servicios. El consumo intermedio es el valor de los bienes y servicios utilizados como insumos en un proceso de producción, los cuales pueden ser transformados o consumidos en el mismo proceso. El valor agregado es el valor adicional creado en el proceso de producción, por lo cual debe ser calculado incluyendo la valoración del consumo de capital fijo como importe de su producción. En conclusión, la producción va ser igual al consumo intermedio menos su valor agregado.

Las artes visuales incluyen las tradicionales artes plásticas, entre ellas, el dibujo, la pintura, el grabado y la escultura, y las expresiones que tienen nuevas tecnologías, donde su mayor componente expresivo es el visual. Las artes escénicas están compuestas por el teatro, la danza, las presentaciones que las articulen, el circo, la pantomima, la narración, la declamación, etc. La educación cultural está compuesta por la no formal, orientada a la lúdica, la formal, haciendo referencia a la primaria y secundaria, la superior, la formación en bellas artes, el diseño y otros, como la curaduría y la gestión cultural.

La creación literaria, musical, teatral y audiovisual representa un sector especial del campo cultural, pues hace parte de todos los sectores de manera transversal, desde la etapa de ideación hasta la puesta en disposición; diversos agentes ejecutan actividades de creación; se tienen en cuenta las obras originales de autores, compositores y otros artistas; se exceptúan artes escénicas, pintores y escultores. Los filmes y videocintas hacen referencia a la producción de películas, cintas cinematográficas, cortometrajes, largometrajes y documentales. La fabricación de juegos-juguetería hace referencia a la producción de todo tipo de juguetes de cualquier material, como madera, cerámica, plástico, caucho, etc., y juegos electrónicos, como videojuegos. La industria musical contiene las presentaciones musicales en vivo, la edición de música y la producción fonográfica. La industria editorial tiene como actividades la producción de libros, la producción de diarios y revistas y de otros trabajos editoriales. Finalmente, la industria de la creación publicitaria contiene los servicios de publicidad y de producciones secundarias.

El porcentaje de la producción es la medición del valor agregado de cada industria sobre su producción total. Los cálculos se hallaron sacando el promedio de los diez años, con su valor mínimo y máximo. Estos datos nos sirven para determinar el nivel de innovación en la producción de bienes y servicios de consumo cultural (Tabla 1). 
Tabla1. Porcentaje sobre la producción durante el periodo 2005-2014

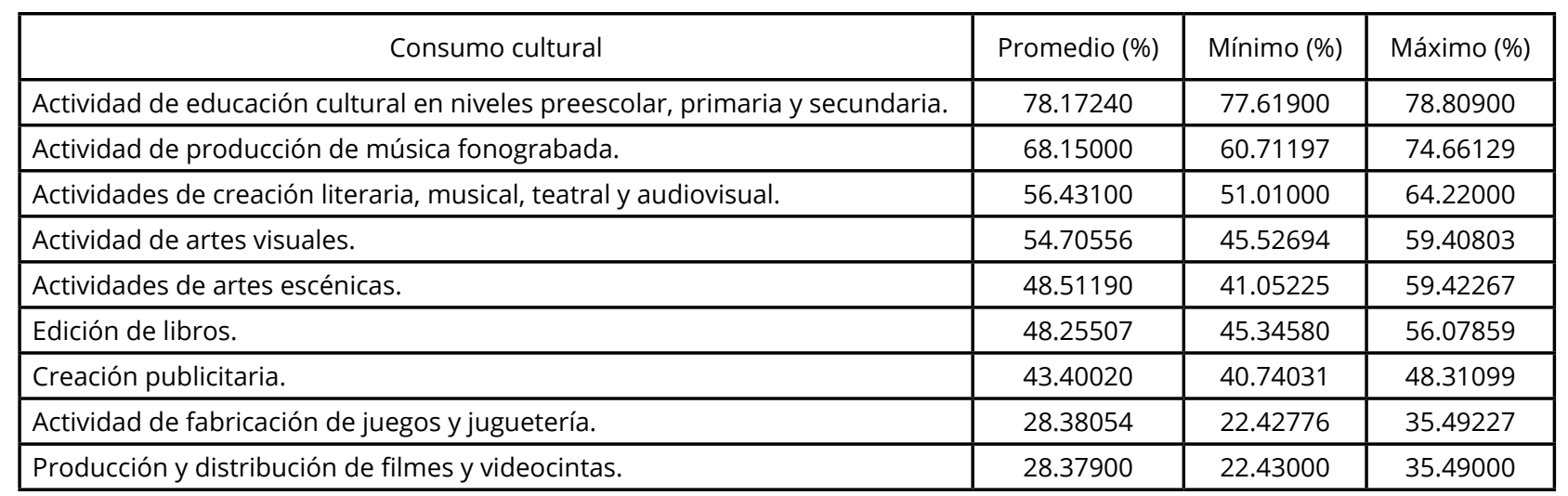

Fuente. Elaboración propia según Cuenta Satélite de Cultura 2005-2016.

La actividad que mayor ponderación tiene es la de educación cultural en niveles preescolar, primaria y secundaria, con un valor de $78.17 \%$, seguido del sector de producción de música, con 68.15 $\%$. Las únicas actividades cuyo valor agregado no representa un porcentaje mayor de $40 \%$ son las actividades de fabricación de juegos y juguetería - $28.38 \%$ - y la producción y distribución de filmes y videocintas $-28.37 \%-$.

Tabla 2. Grado de industrialización durante el periodo 2005-2014
El grado de industrialización es la medición del valor agregado de cada industria sobre el PIB. Los cálculos se hicieron a partir del promedio de este indicador durante el periodo 2005-2014, con su valor máximo y mínimo. Estos datos permiten analizar el comportamiento de las industrias de consumo cultural y su valor agregado en la participación del PIB (Tabla 2).

\begin{tabular}{|c|c|c|c|}
\hline Consumo cultural & Promedio (\%) & Mínimo (\%) & Máximo (\%) \\
\hline Actividad de educación cultural en niveles preescolar, primaria y secundaria. & 0.13866 & 0.12760 & 0.15302 \\
\hline Creación publicitaria. & 0.09515 & 0.08422 & 0.10522 \\
\hline Edición de libros. & 0.08226 & 0.06994 & 0.09848 \\
\hline Actividad de producción de música fonograbada. & 0.01567 & 0.01313 & 0.01840 \\
\hline Actividades de artes escénicas. & 0.01051 & 0.00571 & 0.01512 \\
\hline Actividad de fabricación de juegos y juguetería. & 0.00986 & 0.00690 & 0.01335 \\
\hline Producción y distribución de filmes y videocintas. & 0.00985 & 0.00690 & 0.01330 \\
\hline Actividades de creación literaria, musical, teatral y audiovisual. & 0.00397 & 0.00340 & 0.00440 \\
\hline Actividad de artes visuales. & 0.00090 & 0.00062 & 0.00111 \\
\hline
\end{tabular}

Fuente. Elaboración propia según Cuenta Satélite de Cultura 2005-2016. 
La industria de consumo cultural que aproximadamente presentó un mayor grado de industrialización fue la de educación cultural, en niveles preescolar, primaria y secundaria, con un promedio de $0.1386 \%$, seguido de la industria de la creación publicitaria $-0.09515 \%$ - y la industria editorial -0.08626 \%-. Por su parte, las industrias que aproximadamente tuvieron un menor grado de industrialización fueron las artes visuales -0.0009 \%- y la creación literaria, musical, teatral y audiovisual $-0.00397 \%$ -

Se identifica que en el periodo de 2005-2014 la producción total ha evolucionado, presentando incrementos para los últimos años. La producción se considera como el valor agregado más el consumo intermedio, y establece que la industria de bienes, contenido y servicios culturales se manifiesta como un sector que a largo plazo podría estar aumentando su participación en la producción nacional. Esta alcanza un punto máximo en 2014, representada en aproximadamente COP 3500000 (Figura 1).

Figura 1. Producción, valor agregado y consumo intermedio cultural en Colombia

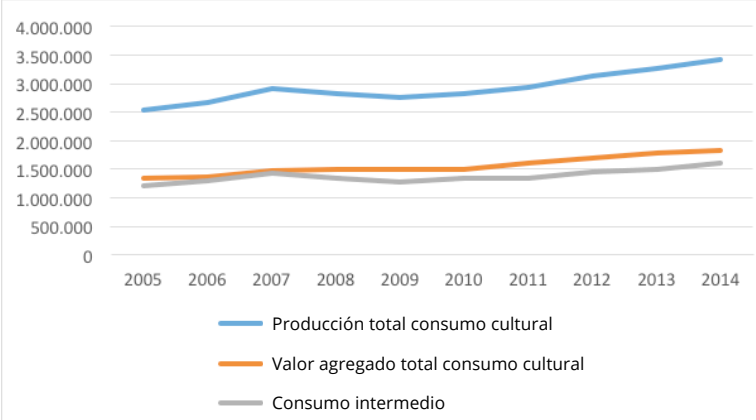

Fuente. Elaboración propia según Cuenta Satélite de Cultura 2005-2016.

El promedio de grado de industrialización del consumo cultural entre 2005 y 2014 es de $0.36 \%$. Se puede determinar que se ha mantenido estable durante estos diez años sin presentar ningún crecimiento o caída desproporcionada. El peso del valor agregado sobre la producción es cercano a la mitad -53.09 \%-; solo ha presentado crecimiento de tres puntos porcentuales desde 2005. La representación sobre PIB, es decir, el valor de la economía naranja, no alcanzó $1 \%$. Su promedio durante los diez años de estudio es de $0.69 \%$, teniendo su valor más alto en 2007 con $0.74 \%$ y su valor más bajo en 2013 con 0.66 \% (Figura 2).
Figura 2. Producción de consumo cultural: aproximación a la economía naranja

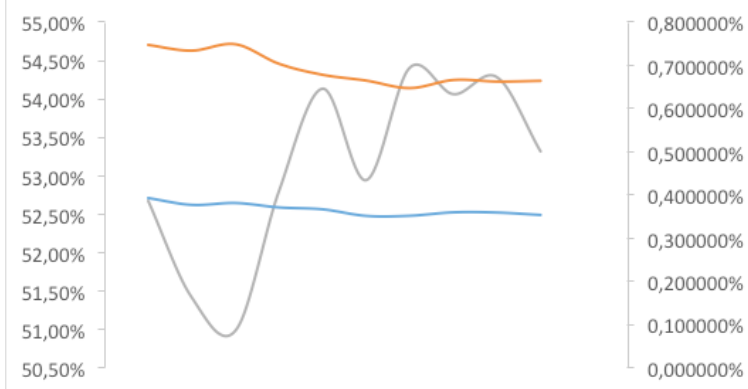

— Porcentaje sobre la producción —_ Grado de industrialización

Representación del PIB

Fuente. Elaboración propia según Cuenta Satélite de Cultura 2005-2016.

\section{Conclusiones}

En cuanto al grado de industrialización, la industria cultural que presentó un mayor porcentaje en el periodo 2005-2014 fueron las actividades de educación cultural, en niveles preescolar, primaria y secundaria, con un promedio de $0.1386 \%$, seguido de la industria de la creación publicitaria $(0.09515 \%$ ). La industria que presentó un menor grado de industrialización durante el periodo estudiado fueron las artes visuales, con un porcentaje de $0.0009 \%$. Se determina que la producción total de la industria de bienes, servicios y contenidos culturales ha presentado crecimiento especialmente para los últimos años, reconociendo que se proyecta como uno de los sectores que puede a largo plazo contribuir en mayor medida a la producción nacional de consumo cultural.

La ponderación del valor agregado sobre la producción de consumo cultural tiene su mayor relevancia en las actividades de educación cultural en niveles preescolar, primaria y secundaria, con un valor de $78.17 \%$, seguido del sector de producción de música, con 68.15 $\%$. Las únicas actividades que su valor agregado no representa un porcentaje mayor de $40 \%$ son las actividades de fabricación de juegos y juguetería y la producción y distribución de filmes y videocintas. Estas últimas deben ser objeto de innovación en los próximos años. 
El peso del valor agregado sobre la producción total de consumo cultural es cercano a la mitad; solo ha presentado crecimiento de tres puntos porcentuales desde 2005 a 2014. El valor de la economía naranja en Colombia de 2005 a 2014 no alcanzó 1 \% ningún año; su promedio fue de $0.69 \%$, valor muy cercano al presentado por otros autores al inicio de la investigación.

\section{Referencias}

Aguado Quintero, L. F. (2010). Estadísticas culturales: una mirada desde la economía de la cultura. Cuadernos de Administración, 23(41), 107-141.

Aguado Quintero, L. F. y Palma Martos, L. (2012). Determinants of performing arts participation in Colombia. Trabajo presentado en 17th International Conference on Cultural Economics by the ACEI, Kioto, Japón.

Aguiar Losada, E. (2014). Las industrias culturales y creativas en España. Working Paper, 13. Recuperado de http://www.uam.es/docencia/degin/ catedra/documentos/13_Las \%20industrias \%20culturales \%20y \%20 creativas \%20en \%20Espana.pdf

Andigraf (Asociación Colombiana de la Industria de la Comunicación Gráfica) (2013). Informe de sostenibilidad 2013: industria editorial y de la comunicación gráfica. Bogotá: Andigraf.

Banco Central de la República Dominicana (2015). Cuenta Satélite de Cultura de la República Dominicana 2015-2016. Recuperado de https:// www.cepal.org/sites/default/files/events/files/2015-semcn-s8repdominicanabc-erosario.pdf

Castro, R., Roldan, J. M., Rodríguez, K., Betancour, P., García, L., Castro, M. y Bohórquez, C. (2006). Diagnóstico económico de los espectáculos públicos de las artes escénicas en Bogotá: teatro, danza, música y circo. Bogotá: Ministerio de Cultura.

DANE (Departamento Administrativo Nacional de Estadística). Cuenta Satélite de Cultura 2005-2016. Recuperado de http://www.dane.gov. co/index.php/estadisticas-por-tema/cuentas-nacionales/cuentassatelite/cuenta-satelite-de-cultura-en-colombia/cuenta-satelite-decultura-2005-2016

DANE (Departamento Administrativo Nacional de Estadística) (2008). COLOMBIA - Encuesta de Consumo Cultural - ECC 2008. Recuperado de http://formularios.dane.gov.co/Anda_4_1/index.php/catalog/107/export

Pis Diez, E. y García, F. (2014). El desarrollo del mercado audiovisual en Argentina: una industria de exportación. Palabra Clave, 17(4), 1137-1167.

Quartesan, A., Romis, M. y Lanzafame, F. (2007). Las industrias culturales en América Latina y el Caribe: desafíos y oportunidades. Washington DC: Banco Interamericano de Desarrollo.

Szpilbarg, D. y Saferstein, E. (2014). De la industria cultural a las industrias creativas: un análisis de la transformación del término y sus usos contemporáneos. Estudios de Filosofía Práctica e Historia de las Ideas, 16(2), 99-112.

Valcerde, E., Salazar, N., Hernández, I. y Ramírez, T. (2015). La economía naranja en Centroamérica. Ciudad de Guatemala: SIECA.

Zuleta J., L. A. y Jaramillo G., L. (2009). Estudio económico de la industria de la música. Bogotá: Ministerio de Cultura. 